\title{
Antecedentes históricos sobre el Complejo Forestal y Maderero Panguipulli, provincia de Valdivia, Centro-sur de Chile
}

\author{
Historical background of the Panguipulli Forestry and Timber Complex, \\ Valdivia province, South-central Chile
}

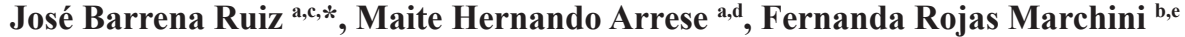 \\ Autor de correspondencia: ${ }^{a}$ Universidad Austral de Chile, Centro Transdisciplinario de Estudios Ambientales \\ y Desarrollo Humano Sostenible, Valdivia, Chile, tel.: 56-63-221915, jose.barrena@uach.cl \\ ${ }^{\mathrm{b}}$ Universidad Austral de Chile, Instituto de Ciencias Ambientales y Evolutivas, Valdivia, Chile. \\ ${ }^{c}$ Universidad de Wageningen, Environmental Policy Group, Wageningen, Países Bajos. \\ ${ }^{d}$ Universidad de Wageningen, Sociology of Development and Change Group, Wageningen, Países Bajos. \\ e Universidad de British Columbia, Departamento de Geografía, Vancouver, Canadá. \\ "Los viejos obreros y sus hijos, apiñados en las poblaciones de Panguipulli y en sus pequeños poblados de Liquiñe, \\ Coñaripe, Llifén, Futrono, Nontuelá, Neltume, Curriñe, sobreviven apenas, mirando las montañas donde otrora \\ vivieron, trabajaron, cantaron, soñaron y amaron" (Pedro Cardyn).
}

\begin{abstract}
SUMMARY
The aim was to inquire on the historical background of Panguipulli Forestry and Timber Complex, a forestry company that emerged in 1971 in the province of Valdivia, South-central Chile. The company managed an area larger than 400,000 hectares and employed over 3,000 workers, functioning under a co-management scheme supported by both workers and state. We carried out a literature review that included scientific articles, books, theses and reports. Additionally, in-depth interviews were conducted with two informants who were involved directly in the historical development of Panguipulli Forestry and Timber Complex. We inquired in both the underlying social processes and direct events that influenced the emergence of the forestry company. A long period of encroachment on indigenous land and the emergence of a rural working-class resulting from the activities of a novel forestry industry fall within the former. While, on the other hand, key political reforms, as well as the coordinated actions led by forestry workers, account for the latter. We argue that the rise of Panguipulli Forestry and Timber Complex is the result of a longstanding attempt by workers and indigenous people to subvert power relations and domination established from the European colonization of the mid-nineteenth century. The research provides relevant information that contributes to the fields of environmental history and local identity, which in turn offer key elements for a current discussion of forestry and rural development.
\end{abstract}

Key words: forestry history, peasant movements, Mapuche people, rural development.

\section{RESUMEN}

El Complejo Forestal y Maderero Panguipulli, empresa surgida en 1971 en la provincia de Valdivia, administró un área superior a 400.000 ha y empleó a más de 3.000 trabajadores bajo un esquema de comanejo entre obreros y Estado. El objetivo fue indagar en los procesos sociales que influyeron en la conformación de esta empresa. Se desarrolló una revisión bibliográfica que incluyó artículos científicos, trabajos de tesis, informes y libros. Adicionalmente, se realizaron dos entrevistas en profundidad a personas que tuvieron directa participación en el proceso histórico de desarrollo del Complejo. Se indagó tanto en los procesos sociales subyacentes como en los acontecimientos que influyeron directamente en su conformación. Dentro de los primeros, destaca un prolongado período de usurpación de tierra indígena impulsado por el Estado de Chile, así como el posterior surgimiento de una población de obreros forestales y madereros a consecuencia de la explotación industrial de la madera en la zona. Por otro lado, importantes reformas políticas así como la articulación de un movimiento campesino-obrero, están entre las causas directas. La investigación plantea que el surgimiento del Complejo Forestal y Madero Panguipulli es el resultado de un prolongado intento de obreros y mapuche por subvertir las relaciones de poder y dominación instauradas a partir de la colonización europea de mediados del siglo XIX. El trabajo, aporta información relevante en los ámbitos de la historia ambiental y la identidad local, entregando elementos de análisis para una discusión actual sobre el desarrollo forestal y rural.

Palabras clave: historia forestal, movimientos campesinos, mapuche, desarrollo rural. 


\section{INTRODUCCIÓN}

La acelerada degradación que experimentan los ecosistemas a escala planetaria como consecuencia de la acción humana, ha impulsado el desarrollo de un creciente número de investigaciones orientadas hacia el estudio de la sustentabilidad socio-ecológica (Anderson et al. 2008, Ostrom 2009). Dentro de este marco, algunos trabajos reconstruyen y analizan la historia ambiental (Moore 2003) y las transformaciones del paisaje (Armesto et al. 2010), con el objetivo de profundizar en el entendimiento de las complejas relaciones entre sociedad y naturaleza. Recientes estudios indican que la modelación de los bosques de la zona Centro-sur de Chile ha sido un proceso dinámico no lineal, influido por diversas formas de asentamiento, estrategias de adaptación y sistemas de gobernanza ambiental desarrollados por poblaciones indígenas, conquistadores españoles, colonos, campesinos, obreros forestales y Estado, durante los últimos 500 años (Camus y Solari 2008, Solari et al. 2011, Lara et al. 2012).

El estudio y descripción de las prácticas de manejo y uso del bosque, es un tema relevante dentro de la historia ambiental y la sustentabilidad de los recursos naturales. En décadas recientes, el enfoque tradicional de manejo de bosques, basado en una estructura vertical centralizada, con estrategias de toma de decisiones que involucran un reducido grupo de actores, ha sido cuestionado por conducir a procesos de degradación ambiental en un horizonte de largo plazo (Hauck et al. 2015, Paletto et al. 2015). Por el contrario, los esquemas que involucran el comanejo de los bosques y la participación de múltiples actores con diversos objetivos, han generado un creciente interés dentro de la investigación científica y los programas de desarrollo impulsados por agencias estatales y organizaciones no gubernamentales, argumentándose que contribuyen a legitimar los procesos de toma de decisiones y reducir los conflictos entre los distintos grupos involucrados (Kangas et al. 2006).

Los bosques generan flujos materiales e inmateriales que sostienen las prácticas culturales y económicas de la población rural en la zona Centro-sur de Chile. Por un lado, las actividades forestales y madereras constituyen los medios de vida para un grupo heterogéneo de familias rurales, mientras la producción industrial de gran escala genera miles de puestos de trabajo transitorios, los que a su vez movilizan fuerza de trabajo y tecnología. Por otro lado, las comunidades mapuche ${ }^{1}$ han mantenido una larga convivencia con los bosques templados, desde los que obtienen alimentos, medicina y leña, y en los que reconocen sitios sagrados para la realización de prácticas ceremoniales (Herrmann 2006).

\footnotetext{
Se usa la palabra mapuche tanto en singular como en plural respetando el uso que los/as mismos/as mapuche hacen de la palabra. Como indica Marimán (2012) el componente che da la pluralidad a la palabra, de modo que resulta redundante decir mapuches así como sería decir gentes en castellano.
}

La sustentabilidad ecológica del bosque depende de las prácticas sociales involucradas en su uso, así como de las normas e instituciones formales e informales que regulan los intereses de personas y grupos sociales relacionados con esas prácticas. Los "usuarios" del bosque, sostienen distintos valores y persiguen diversos intereses, los que intentan promover mediante el funcionamiento de instituciones sociales como el mercado, el sistema de propiedad privada y el Estado. La actuación de estos grupos, así como la conformación y cambio de las instituciones sociales dentro de la producción forestal y maderera, son temas relevantes desde un punto de vista político, cultural, ecológico y económico. No obstante, los estudios sociales sobre el bosque continúan siendo escasos.

En este contexto, esta investigación indaga en los procesos sociales que influyeron en la conformación del Complejo Forestal y Maderero Panguipulli (COFOMAP), empresa surgida en 1971 en la provincia de Valdivia, Centro-sur de Chile, que administró un área superior a las 400.000 ha mediante un esquema de comanejo en el que obreros y Estado decidían conjuntamente sobre la producción, comercialización y uso de los recursos del bosque (CODEPU 1991, Rivas 2006, Pino y Cardyn 2014). Además de las contingencias relacionadas a las reformas y procesos político-sociales iniciados en la segunda mitad de la década de 1960, la formación del COFOMAP también reúne procesos históricos subyacentes, vinculados a la configuración de la propiedad rural desde las últimas décadas del siglo XIX, y al posterior surgimiento de una clase obrera rural en la década de 1940, con el inicio de la producción industrial de madera en la zona.

La integración de estos procesos sociales en una narración histórica, entrega una perspectiva de los cambios en las relaciones de poder y las estructuras de dominación en la producción forestal y maderera de la zona centro-sur de Chile. Por un lado, el poder es la capacidad relacional que permite a un actor social influir sobre las decisiones de otros actores, con el objetivo de favorecer su propia voluntad, intereses y valores, mientras que, por otro lado, la dominación corresponde al poder enquistado en las instituciones sociales (Castells 2009). En este sentido, esta investigación plantea que el COFOMAP es el resultado de un prolongado intento de obreros e indígenas por subvertir las relaciones de poder y dominación instauradas a partir de la colonización europea de mediados del siglo XIX.

El presente trabajo corresponde a una revisión y análisis de la literatura existente sobre el COFOMAP, la que es ampliada con otras fuentes bibliográficas relacionadas con la historia ambiental y las prácticas sociales involucradas en el uso de los recursos naturales, particularmente en relación al bosque. Adicionalmente, se realizaron dos entrevistas en profundidad (informante 1, informante 2), durante los meses de noviembre y diciembre 2010. El informante 1 fue militante del Movimiento de Izquierda Revolucionaria (MIR) desde 1968, y entró en contacto con la zona de Neltume en 1969, participando junto a obreros en tomas de 
fundos previo a la conformación del COFOMAP, así como también en la organización y funcionamiento de esta empresa hasta el golpe de Estado de 1973. El informante 2, se desempeñó como encargado del Departamento de Manejo de Bosques del COFOMAP.

\section{DESPOSESIÓN MAPUCHE Y COLONIZACIÓN EUROPEA}

Durante las primeras décadas del siglo XIX, las recientemente conformadas naciones de Argentina, Chile y México, realizaron incursiones militares dentro de lo que concebían como sus espacios nacionales con la finalidad de ampliar sus fronteras de producción agrícola. Frecuentemente, estas incursiones culminaron con la colonización de territorios indígenas, despojando a sus habitantes del libre uso de la tierra (Aylwin 2002). En Chile, el Estado invadió militarmente (1861) los asentamientos mapuche establecidos al sur del río Biobío, lugar acordado como frontera entre los territorios ocupados por los conquistadores españoles y el pueblo mapuche en el parlamento de Quilín, celebrado el 5 y 6 de enero de 1641 (Bengoa 2007). La invasión y posterior ocupación provocó numerosas muertes entre la población mapuche, tanto de los enfrentamientos y masacres, como por el surgimiento de nuevas enfermedades (Bengoa 2000).

Consumada la victoria, se inició un proceso de delimitación de la propiedad indígena y asentamiento de colonos, para lo cual el 4 de diciembre de 1866, se dictó la ley sobre fundación de ciudades en los territorios indígenas al sur del río Biobío (Almonacid 2009, Correa y Mella 2010). Por medio de esta ley, se fijaron los terrenos ocupados "efectiva y continuamente" por los indígenas, sin considerar los espacios de pastoreo, ramoneo, extracción de leña y recolección de frutos utilizados ancestralmente por las comunidades mapuche. Estos espacios fueron considerados terrenos "baldíos", siendo posteriormente rematados y entregados a colonos nacionales y extranjeros (Correa y Mella 2010).

Como resultado, los mapuche fueron confinados en reducciones, entregándose títulos colectivos de propiedad - conocidos como títulos de merced - a las autoridades o representantes de las comunidades radicadas (Foerster 1996, Di Giminiani 2015). Solo a partir de 1927, con la promulgación de la ley de división de comunidades indígenas, el Estado impulsó un proyecto de "integración" forzosa de los indígenas a la sociedad chilena como propietarios individuales. Entre 1884 y 1929 alrededor de 90 mil mapuche fueron ubicados en cerca de tres mil reducciones (Almonacid 2008), mientras que 30 mil a 40 mil permanecieron fuera de las radicaciones, especialmente desde la provincia de Valdivia al sur (Foerster 1996, Bengoa 2000, Correa et al. 2005). Al final del proceso de radicación, el Estado confinó a la población mapuche dentro de un espacio equivalente al $5 \%$ de su territorio original (Di Giminiani 2015).

En la provincia de Valdivia, la colonización europea se inició con anterioridad a la invasión militar de la década de 1860. En 1846 un grupo de familias alemanas arribó al puerto de Corral ayudadas por el agente colonizador del Estado de Chile Bernardo Philippi, quien en los años siguientes conduciría el asentamiento de más de 200 familias alemanas en la provincia de Valdivia, atraídas con el ofrecimiento de semillas, ganado y tierra a bajo costo (Vega 1958). Los colonos desarrollaron actividades de ganadería, agricultura comercio e industria. En 1898 entra en funcionamiento la empresa forestal "Camino Lacoste i Compañía", mientras en 1906 se establece la Compañía Ganadera San Martín, la que introdujo el primer barco a vapor en el lago Panguipulli (Rivas 2006).

$\mathrm{El}$ asentamiento de colonos previo a la radicación mapuche en la provincia de Valdivia, generó exiguas posibilidades de constituir reducciones debido a la escasez relativa de tierras (Almonacid 2009). No obstante, solo en la comuna de Panguipulli se establecieron 193 reducciones, que a comienzos del siglo XX tenían un promedio de 10 ha por persona. Diversas leyes sobre la propiedad de la tierra dictadas entre 1925 y 1931, no evitaron la continua usurpación de tierra indígena, la que ocurría a través de la adquisición de acciones y derechos de propiedad por parte particulares no indígenas, quienes una vez concretadas las ventas, extendían violentamente los límites de las tierras adquiridas (Almonacid 2008). La conformación de la propiedad rural se desarrolló bajo constantes abusos sobre la población indígena, quienes sufrieron desalojos y fueron en muchos casos engañados para traspasar sus propiedades a colonos, lo que ocurrió con la participación de autoridades nacionales y regionales (Almonacid 2009). A comienzos de la década de 1970, la superficie de tierra había caído abruptamente a 1,7 ha por persona en algunas zonas de la comuna de Panguipulli (Le Bonniec 2013).

El 18 de enero de 1907, con el objeto de informarse y buscar soluciones sobre los atropellos y abusos cometidos contra los mapuche, se celebró el parlamento indígena de Koz-koz, al que asistieron gran cantidad de Lonko ${ }^{2}$ asentados en los territorios delimitados por Purulón (Oeste), la república de Argentina (Este), la ciudad de Villarrica (Norte) y Panguipulli (Sur) (Díaz 2005). Durante el parlamento, Juan Catriel Rain, Lonko principal de Trailafquén, fue elegido como representante de los mapuche para establecer diálogo con las autoridades nacionales y explicar así los problemas que enfrentaban los indígenas de la zona. El 25 de enero de 1907, el misionero capuchino Sigifredo de Frauenhäusl, en representación del Lonko Catriel, envió una carta al ministro de Relaciones Exteriores, Culto y Colonización, en donde expuso cada uno de los abusos que se cometían contra los mapuche, entre los que se incluían usurpaciones de terrenos, quemas de casas, golpizas, violaciones y asesinatos (Díaz 2005).

A pesar de las permanentes denuncias realizadas frente a las autoridades nacionales y locales, el abuso sobre la

Corresponden a los jefes tradicionales de un grupo familiar (Lof), que heredan su cargo por linaje. 
población indígena continuó durante el siglo XX. La colonización extranjera y nacional, otorgaba un carácter civilizador que el Estado pretendía imponer sobre la población indígena y, al mismo tiempo, constituía una estrategia que permitía la expansión de la soberanía nacional hacia los territorios del sur (Correa y Mella 2010). Desde la perspectiva de la oligarquía gobernante, la inmigración europea contribuía notablemente a impulsar la industria, el comercio y las artes (Domeyko y Förster 1850). En este contexto, se promulga en 1931 el decreto supremo $\mathrm{N}^{\circ} 4363$, conocido como Ley de bosques, mediante el cual se estableció la aptitud forestal de los terrenos, se fomentó la forestación y se contemplaron recursos y exenciones tributarias para incentivar la producción forestal (Chacón 1994).

\section{PRODUCCIÓN FORESTAL Y PROLETARIZACIÓN CAMPESINA}

A partir del proceso colonizador se inicia un creciente desarrollo industrial en la provincia de Valdivia, el que incluyó la instalación de aserraderos y el desarrollo de un activo mercado de la tierra entre las últimas décadas del siglo XIX y la primera mitad del siglo XX (Almonacid 2009). Durante la década de 1930, la instalación de empresas agrícolas y forestales provocó una importante inmigración de fuerza de trabajo hacia la zona. En 1939 se constituye la Sociedad Agrícola y Maderera Neltume Limitada, a la cual Berenice Aravena e Hijos aportan el fundo Neltume, predio de 36.449 ha de extensión (Rivas 2006).

En 1942, la Sociedad Agrícola y Maderera Neltume Limitada inaugura una fábrica para la producción de madera terciada. Posteriormente, la misma sociedad instaló la Industria de Maderas Sociedad Anónima (IMASA), donde se fabricaban puertas, ventanas, marcos y molduras (Bravo 2012). La operación de estas fábricas trajo nuevas inmigraciones hacia la zona de Neltume, empleándose cerca de 500 trabajadores sólo en la fábrica de terciados, quienes cumplían turnos de 12 a 14 horas diarias. La fábrica funcionaba las 24 horas del día, y el pago de los obreros se entregaba en vales o fichas, los que sólo podían cambiarse por productos de la pulpería patronal (Galilea et al. 1972, Bravo 2012).

Las condiciones precarias en que se desarrollaba la vida del obrero forestal, la falta de dinero y el aislamiento, motivan tomas y huelgas. En el fundo Carranco, cercano a Neltume, se produjeron tomas en los años 1944 y 1953, las que fueron violentamente reprimidas por la policía (CODEPU 1991). En 1951, los trabajadores de la Fábrica Neltume, exigiendo mejores condiciones habitacionales y laborales, levantaron una huelga que duró tres meses. La movilización fue reprimida, sus dirigentes fueron detenidos y encarcelados en la ciudad de Valdivia; la mayor parte de los trabajadores participantes fueron despedidos y la empresa se dotó de un contingente especial de carabineros para reprimir cualquier nuevo intento de huelga (Rivas 2006).
Con la llegada del ferrocarril, en 1954, la producción maderera creció notablemente en la zona de Panguipulli y Neltume. Para el año 1961, en la provincia de Valdivia se producían 5.736 .000 pulgadas de madera $\left(135.350 \mathrm{~m}^{3}\right)$ y existían 136 aserraderos, lo que la ubicaba como la segunda provincia en volumen de producción y la primera en número de aserraderos a escala nacional (Rivas 2006). Las principales especies forestales utilizadas en la producción de madera eran el ulmo (Eucryphia cordifolia Cav.), la tepa (Laureliopsis philipiana (Looser) Schodde.), el raulí (Nothofagus alpina (P. et E.) Oerst.) y el roble pellín (Nothofagus obliqua (Mirb) Oerst.).

Acontecimientos de carácter nacional e internacional impulsaron transformaciones en la estructura de la sociedad rural chilena durante la década de 1960 (Kay y Pineda 1998). En 1962 el gobierno conservador de Jorge Alessandri (1958-1964) aprobó la primera ley de reforma agraria (Ley 15020) influido fuertemente por la política exterior de los Estados Unidos, país que impulsó reformas agrarias redistributivas en América Latina como una forma de contener posibles estallidos sociales y el avance del comunismo en la región (Bellisario 2013). La ley incluyó la creación de la Corporación de Reforma Agraria (CORA), organismo estatal encargado de la planificación y ejecución de la reforma, aunque su utilización fue limitada durante el gobierno de Alessandri, otorgándose tierra a sólo 1.100 campesinos, correspondiente a poco más del $10 \%$ de lo planificado para el final del gobierno (Thiesenhusen 1966).

Durante los seis años siguientes, el gobierno Demócrata Cristiano de Eduardo Frei (1964-1970), implementó un programa de reforma agraria orientado a: 1) mejorar la productividad y producción, para lo cual se planificó la expropiación de latifundios explotados de forma ineficiente, 2) mejorar las condiciones de vida en zonas rurales, mediante la entrega de tierra a 100.000 familias campesinas y 3) aumentar la base de apoyo al partido Demócrata Cristiano, mediante la sindicalización campesina bajo el patrocinio del gobierno. Si bien la productividad agrícola aumentó significativamente y los campesinos sindicalizados pasaron de unos pocos miles a cerca de 140.000 en 1970, sólo le fueron entregadas tierras al $7 \%$ de las 100.000 familias proyectadas (Kay 1977).

Durante el año 1965, el gobierno envió al congreso el proyecto de una nueva ley de reforma agraria (Ley 16640), la que fue promulgada en 1967. Ese mismo año, se dicta la ley sobre sindicalización campesina (Ley 16625), que en el ámbito local favoreció tanto la creación del Sindicato Agrícola e Industrial de la Sociedad Agrícola y Maderera Neltume Limitada, como la Federación Provincial Campesina Indígena Ranquil, que agrupó a más de 28.000 campesinos e indígenas de toda la provincia de Valdivia (Rivas 2006).

La conformación de organizaciones obreras y campesinas, así como la existencia de un clima político favorable para las reivindicaciones de la clase proletaria, cimentaron las condiciones para los profundos cambios venideros. En- 
tre 1964 y 1973, el movimiento campesino chileno alcanzó una relevancia nacional nunca antes experimentada, la cual no recuperó posteriormente (Gómez 2002). En este contexto, en diciembre de 1968, un grupo de estudiantes de la Universidad Austral de Chile concurrió a desarrollar trabajos y prácticas profesionales en el fundo Trafún. La experiencia permitió a los estudiantes conocer las condiciones de vida y trabajo de los obreros forestales, y sería la primera de una serie de colaboraciones a las que se sumarían estudiantes de las universidades de Chile, Concepción y Técnica del Estado (Bize 2012). Varios jóvenes eran militantes del MIR, grupo de ideología marxista abocado a la construcción colectiva del poder popular, para lo cual impulsaba el control obrero sobre los medios de producción, la distribución de las mercancías y la repartición de la riqueza nacional (Rivas 2006, Bize 2012). Los jóvenes del MIR tomaron contacto con indígenas y obreros y apoyaron tomas de fundos en la zona (informante 1). La existencia de una masa obrera semi-industrial, así como las condiciones topográficas del lugar, fueron fundamentales para la focalización del trabajo del MIR en la zona (Cardyn 2006, informante 2).

\section{CONFORMACIÓN DEL COFOMAP}

Apoyado por los partidos de la Unidad Popular, Salvador Allende logra el triunfo en las elecciones presidenciales de septiembre de 1970. Dentro de su programa de gobierno, la Unidad Popular planteaba la intensificación del proceso de reforma agraria por medio de políticas que fomentaran la aceleración de las expropiaciones, la generación de cooperativas campesinas y la devolución de tierra a las comunidades mapuche (Unidad Popular 1970). En noviembre del mismo año, los obreros del fundo Carranco iniciaron una toma, que sería la cuarta en la historia del fundo (CODEPU 1991). El hecho fue conocido como El Grito de Carranco y constituyó el inicio de una sucesión de tomas en la precordillera y cordillera de las comunas de Panguipulli, Lago Ranco, Los Lagos, Río Bueno y Futrono, desarrolladas por la acción coordinada de obreros forestales y militantes del MIR. Fueron tomados los fundos Pilmaiquén, Huilo Huilo, Arquilhue y Neltume, entre otros (Rivas 2006, Bravo 2012). La acción del movimiento obrero incluyó tanto el control de la tierra como de las instalaciones (galpones, lecherías, bodegas) y capital de trabajo existente (maquinaria, vehículos, animales), lo cual marcó un aspecto diferenciador respecto de otras tomas ocurridas en el marco de la reforma agraria (informante 2, Le Bonniec 2013).

Durante la Unidad Popular, diversas organizaciones obreras e indígenas utilizaron las tomas como una medida de presión para impulsar al gobierno a realizar la expropiación legal de los fundos (Le Bonniec 2013). Durante los primeros dieciocho meses de gobierno se ocuparon ilegalmente aproximadamente 1.500 latifundios (Kay 1974), mientras que durante todo el gobierno de la Unidad Po- pular fueron expropiados 4.490 predios correspondientes a 6,6 millones de hectáreas (Chonchol 1994). Lo anterior fue liderado a nivel nacional por la provincia de Valdivia, que en 1971 contaba con más de 151 fundos tomados (Klein 1973).

El mismo año, los obreros del fundo Neltume completaron más de dos meses en toma sin lograr acuerdo con sus dueños, por lo que el sindicato solicitó al gobierno la intervención y posterior expropiación del fundo (Rivas 2006). El gobierno accedió a esta demanda y decidió la expropiación de 14 predios en la cordillera y precordillera de la provincia de Valdivia, lo que fue conocido como la Operación Ardillas (CODEPU 1991). El posterior análisis de las escrituras e inscripciones de estos fundos, evidenció la larga historia de usurpación y engaños que ocultaba el proceso de colonización iniciado a mediados del siglo XIX (Rivas 2006).

El 15 de abril de 1971, a través del acuerdo número 615 de la CORA, se decide transferir los predios expropiados a la Corporación de Fomento de la Producción (CORFO), organización del Estado creada en 1939 con el objetivo de impulsar la producción e industria nacional (Galilea et al. 1972). Durante los meses siguientes, obreros, gobierno, MIR y otros movimientos políticos con presencia en la zona, planificaron la conformación de una gran empresa forestal dirigida por los trabajadores (informante 1, informante 2). El 17 de octubre se creó el COFOMAP, formado por la sociedad entre CORFO y forestal Pilpilco, empresa subsidiaria de CORFO, creada en 1967. En un comienzo, la superficie del COFOMAP alcanzó poco más de 300.000 ha, pero posteriormente, nuevas expropiaciones y traspasos sumaron más predios, llegando a una superficie total cercana a las 420.000 ha, convirtiéndose en la empresa forestal más grande de Chile, con un área equivalente al $27 \%$ de toda la provincia de Valdivia (CODEPU 1991) (figura 1).

La nueva fase de la reforma agraria iniciada durante el período de la Unidad Popular, otorgó tanto a mapuche como a obreros un espacio para la concreción de sus demandas. No obstante, la búsqueda de distintos fines generó diversos grados de tensión y conflicto. Mientras por un lado las comunidades mapuche se movilizaban por la restitución de sus tierras usurpadas, por otra parte la clase obrera se activaba para tomar el control de la tierra y los medios de producción. En alguna medida, el control obrero de los fundos representaba una nueva forma de usurpación y explotación para los mapuche, lo que llevó a que algunas comunidades reivindicaran sus derechos ancestrales sobre la tierra con tomas en predios previamente expropiados por la CORA y ya bajo el control de los trabajadores. De acuerdo con Le Bonniec (2013), después de la conformación del COFOMAP las comunidades indígenas Juan Quintuman, Valeriano Cayicul, Cachim, Fidihuincul y Liquiñe presentaron situaciones de conflicto con la administración obrera recientemente constituida, las que fueron registradas y tratadas por la Dirección de Asuntos Indígenas Nacional (DASIN). 


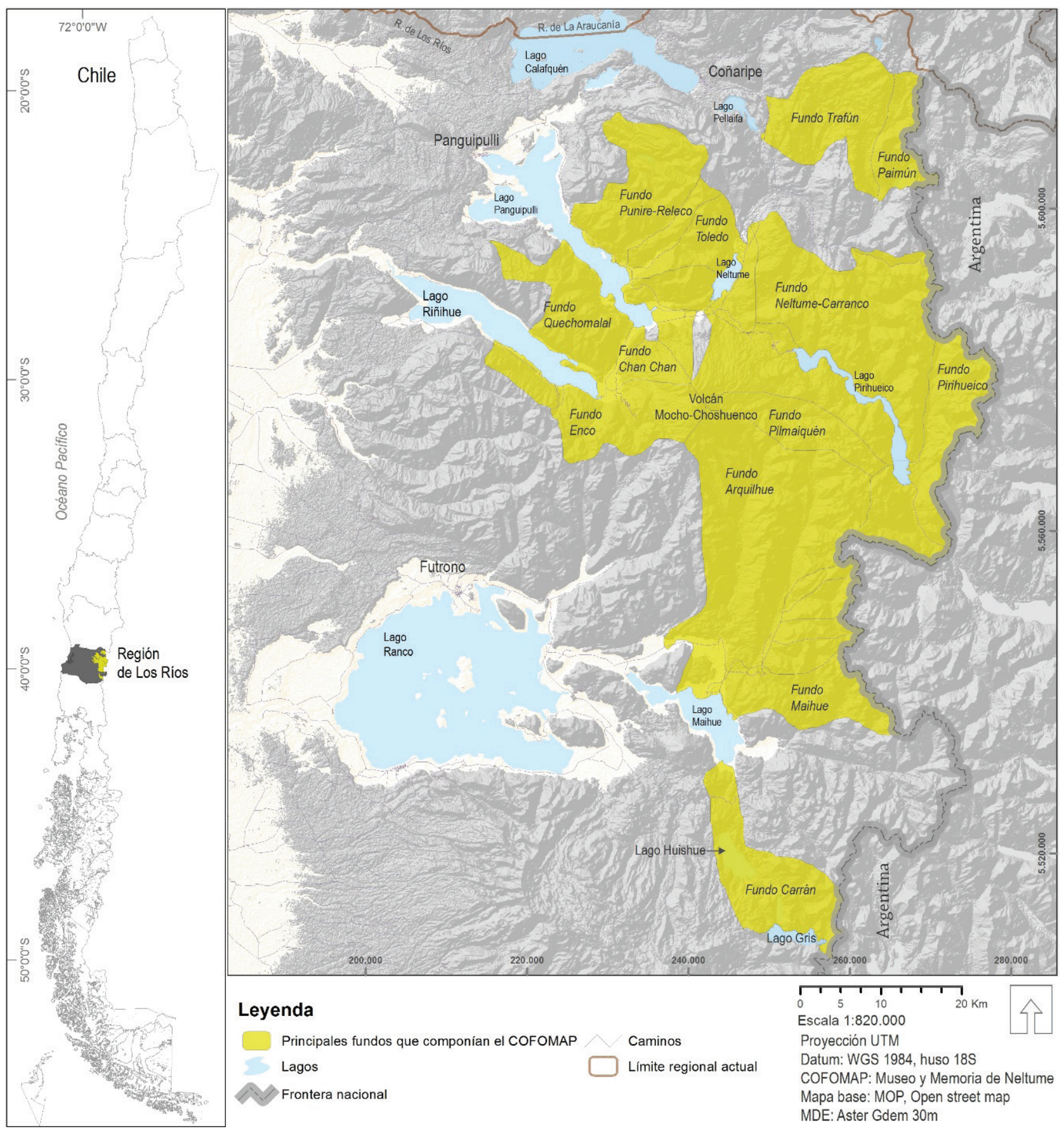

Figura 1. Complejo Forestal y Maderero Panguipulli y sus fundos principales.

Panguipulli Forestry and Timber Complex and its main estates. 


\section{ORGANIZACIÓN Y PRODUCCIÓN DEL COFOMAP}

Los predios que constituían el COFOMAP fueron agrupados en siete sectores. En cada uno ellos los trabajadores elegían un jefe de predio y un jefe de sector (informante 2). Adicionalmente, los obreros elegían un representante para integrar el Consejo de Administración, organismo de mayor importancia en la estructura jerárquica del COFOMAP, el que era además constituido por dos delegados del gobierno (Rivas 2006). Neltume, donde operaban aserraderos y fábricas de madera hacía ya tres décadas, constituyó el sector más importante dentro del COFOMAP, concentrando cerca de un $20 \%$ del total de trabajadores. Otros fundos de importancia en relación al número de obreros, fueron Puñire-Releco y Quechomalal (cuadro 1).

Los principales objetivos trazados por la administración del COFOMAP fueron: 1) la explotación forestal y maderera de los recursos de su propiedad o de terceros; 2) la conservación y la preservación de los recursos na- turales del área geográfica bajo su administración; y 3) la comercialización, ventas y exportación de su producción (CODEPU 1991). Para el desarrollo de sus actividades, el COFOMAP contaba con los departamentos de manejo de bosques, aserraderos, camiones y transporte, mantención y reparación de equipos, clasificación de inventario y despachos, y actividades agropecuarias. Los tres primeros formaban la sub-dirección de explotación de recursos naturales, mientras que los tres últimos integraban la subdirección de recursos industriales (Rivas 2006).

La característica más importante dentro de la organización del COFOMAP, fue la participación de los trabajadores en los distintos niveles de administración, dirección y control de la producción, siendo cualquier decisión de importancia tomada colectivamente a través de asambleas que reunían a obreros, técnicos y profesionales (CODEPU 1991, informante 2). No obstante, la toma de decisiones estratégicas se volvió frecuentemente un proceso complejo debido a la alta presencia de partidos y movimientos políti-

Cuadro 1. Superficie y número de trabajadores de los predios pertenecientes al COFOMAP en 1972. Area and number of workers in the estates that belonged to COFOMAP in 1972.

\begin{tabular}{clcc}
\hline Número del predio & Nombre del predio & Superficie (ha) & Número de trabajadores \\
\hline 1 & Arquilhue & 36.572 & 150 \\
2 & Carranco & 28.000 & 70 \\
3 & Carrán-Hishue & 18.580 & 50 \\
4 & Caulle & 24.739 & 50 \\
5 & Chan-Chan & 6.910 & 24 \\
6 & Enco 1 & 4.083 & 117 \\
7 & Enco 2 & 8.500 & $\mathrm{~s} / \mathrm{d}$ \\
8 & Heinahue & 4.200 & 100 \\
9 & Maihue 1 & 2.080 & $\mathrm{~s} / \mathrm{d}$ \\
10 & Maihue 2 & 8.777 & 100 \\
11 & Maihue 3 & 5.297 & $\mathrm{~s} / \mathrm{d}$ \\
12 & Neltume & 8.403 & 470 \\
13 & Paimún & 4.131 & 47 \\
14 & Pilmaiquén & 33.542 & 130 \\
15 & Pirihuico & 16.886 & 80 \\
16 & Puñire-Releco & 20.519 & 270 \\
17 & Quechomalal & 14.306 & 192 \\
18 & Rudemeica & 3.000 & 100 \\
19 & Ruñinahue & 16.000 & 100 \\
20 & Toledo & 7.214 & 120 \\
21 & Traful & 12.500 & 100 \\
22 & Trafún & 20.500 & 140 \\
\hline Total & & 304.739 & 2.410 \\
\hline
\end{tabular}

Fuente: Galilea et al. (1972). s/d: sin dato. 
cos al interior del COFOMAP, lo que a su vez generó tensiones con el gobierno, el cual consideraba que la actividad política materializada en extensas asambleas y reuniones, conducía al descuido de los aspectos productivos de la empresa (Rivas 2006). Mientras por un lado, algunos sectores planteaban continuar con la toma de predios con el objetivo de incrementar la riqueza y medios de producción en manos de los obreros, otros sectores concentraban esfuerzos en aumentar la producción de los predios que ya se encontraban en poder de los trabajadores (informante 1).

En relación con aspectos productivos, en los primeros años de la década de 1970 la provincia de Valdivia contaba con más de un millón de hectáreas de uso forestal, de las cuales alrededor de un $70 \%$ eran potencialmente productivas, mientras el $30 \%$ restante se consideraban aptas para fines de conservación (Morales et al. 1977). El COFOMAP contaba con cerca de 160.000 ha de bosque nativo, que representaban un volumen total aserrable de alrededor de 520 millones de pulgadas madereras $\left(12.270 .500 \mathrm{~m}^{3}\right)$, concentrando un $41 \%$ del total del recurso maderero aserrable de toda la provincia de Valdivia (Morales et al. 1977). Particularmente, la zona de desarrollo del COFOMAP generaba más de la mitad de toda la producción nacional de madera aserrada de especies nativas, mediante la explotación de especies como el roble y raulí. Contreras et al. (1996), estiman que durante el funcionamiento del COFOMAP se plantaron en el área de Panguipulli entre 3.000 y 4.000 ha de esta última especie, mientras Galilea et al. (1972) indican que el volumen de exportación de raulí desde la zona del COFOMAP, superaba el $80 \%$ del total exportado de la especie a nivel nacional. A fines de 1971, se realizó volteo por cinco millones de pulgadas madereras $\left(118.000 \mathrm{~m}^{3}\right)$, exportándose 28.000 pulgadas de madera $\left(660 \mathrm{~m}^{3}\right)$ a Cuba y $53.000\left(1.250 \mathrm{~m}^{3}\right)$ a Argentina (CODEPU 1991).

Las actividades del COFOMAP dinamizaron la producción y el empleo del sector forestal de la provincia de Valdivia. Alrededor de 3.000 obreros se establecieron en torno a las actividades de producción del COFOMAP, lo que sumado a sus familias superó las 7.000 personas (Galilea et al. 1972, Rivas 2006). El COFOMAP duplicó el empleo existente previamente en los fundos que lo componían, otorgando puestos de trabajo estable a más de 1.000 cesantes e incorporando a un buen número de indígenas de las comunidades cercanas (Rivas 2006). A nivel de la unidad de producción familiar, el ingreso obtenido por el obrero era complementado con distintas actividades agrícolas y forestales, tanto con fines de subsistencia como comerciales, entre las que destacaban la producción de leña, la recolección de productos forestales no maderables y la crianza y caza de animales (Klubock 2004).

\section{DESINTEGRACIÓN DEL COFOMAP}

El proceso de cambios productivos y sociales impulsado por los trabajadores forestales y el gobierno de la Unidad Popular, se ve bruscamente interrumpido con el golpe de
Estado de septiembre de 1973. La zona del COFOMAP es especialmente reprimida por el ejército golpista, siendo allanados todos los predios y caseríos ubicados dentro del área. Existen aproximadamente 87 casos de violación a los derechos humanos con resultado de muerte o desaparición en la provincia de Valdivia, de los cuales 71 ocurrieron durante los tres meses posteriores al golpe de Estado. De esas personas, 44 desempeñaron labores en los sectores de Trafún, Neltume y Arquilhue al interior del COFOMAP (CODEPU 1991). Luego del golpe de Estado, un grupo de obreros y militantes del MIR intentaron resistir en la zona cordillerana de Neltume, pero fueron detenidos, torturados y posteriormente fusilados los primeros días de octubre de 1973.

Con el control del COFOMAP, el gobierno militar decidió realizar una evaluación de su situación, encargando esta misión al Coronel Delegado René López García, quien con fecha 8 de enero de 1974 emite un informe titulado "Breve Síntesis del Complejo Forestal y Maderero Panguipulli Ltda. (COFOMAP)" (Rivas 2006). En dicho informe, el Coronel López destaca la existencia de una estructura orgánica operable y un nivel de producción por sobre los niveles trazados para el año 1973. Además, resalta los alcances del programa agropecuario diseñado por los obreros, el que incluía la producción de alimentos para la población, un manejo y alimentación adecuados para la masa ganadera y la participación de la empresa en los programas productivos desarrollados a escala nacional (CODEPU 1991).

Luego de describir la existencia de una serie de proyectos en relación a las metas futuras del COFOMAP, el Coronel López concluye que: 1) El COFOMAP debe continuar en manos del Estado, por las posibilidades económicas y sociales que representa para la región y el país, 2) el Estado es la única entidad que asegura las mejores condiciones y posibilidades de manejo de las riquezas que ofrece el COFOMAP, y 3 ) la incorporación de nuevas industrias o inversiones en el área, no pueden cambiar la política de unidad del COFOMAP en manos del Estado (CODEPU 1991).

El gobierno decidió que el COFOMAP fuera transferido a la Corporación Nacional Forestal (CONAF), institución de derecho privado creada en abril de 1973 con el fin contribuir a la conservación, incremento, manejo y aprovechamiento de los recursos forestales del país, la cual posteriormente pasó a depender de CORFO. Bajo esta estructura, el $95 \%$ de las tierras fueron traspasadas directamente a CORFO y el $5 \%$ restante a la Sociedad Agrícola SACOR, filial de CORFO (CODEPU 1991). Posteriormente, y a pesar de la positiva evaluación hecha por el Coronel Delegado y la recomendación de mantener la unidad productiva del COFOMAP en manos del Estado, CORFO inicia la licitación de las tierras del COFOMAP, con lo que comienza también su desintegración, que culminó a fines de la década de 1980. Todo este proceso generó cesantía y pobreza en la zona, razón por la cual un gran número de obreros y sus familias emigraron hacia la ciudad de Panguipulli y otras localidades cercanas (Cardyn 2006). 
A fines de la década de 1970, un grupo de militantes del MIR y antiguos obreros del Complejo en el exilio, trazan la Operación Retorno o Plan 78, que consistió en el regreso clandestino a Chile de una docena de militantes para incorporarse a la resistencia contra la dictadura militar (Comité Memoria de Neltume 2003). En 1980, parte de ellos se interna en la cordillera en la zona de Quelhuenco, Tregua, Liquiñe Alto y Alto Pasa, conformando el Destacamento Guerrillero Toqui Lautaro (DGTL). Los guerrilleros logran establecerse en la cordillera, hasta que en junio de 1981 son descubiertos por una patrulla del ejército. A pesar de ello, logran evadir el cerco militar y resistir por varios meses en precarias condiciones en la montaña. Finalmente, entre septiembre y diciembre de 1981, tres integrantes del DGTL fueron detenidos, torturados y asesinados, mientras otros seis murieron en combates o emboscadas (Comité Memoria de Neltume 2003, Cardyn 2006).

\section{DISCUSIÓN Y CONCLUSIONES}

El COFOMAP representa la concreción de las reivindicaciones de los trabajadores forestales en términos de mejoras laborales y sociales. Pero por sobre todo, representa su determinación para establecer la autogestión en el uso de los recursos naturales de su territorio. El proyecto trazado por el COFOMAP superaba los aspectos netamente económicos, integrando elementos sociales y ecológicos, lo que incluía sostener la alimentación de la población a través del desarrollo de un programa agropecuario, programas de vivienda y educación para los trabajadores y sus familias, y la instalación de viveros y prácticas de reforestación con especies nativas (Pino y Cardyn 2014).

El golpe de Estado de 1973 y la consiguiente intervención militar del COFOMAP, acabó con la alianza entre obreros y Estado pactada para llevar a cabo la dirección de la empresa e inició un período de profundos cambios para el sector forestal en particular, y la sociedad chilena en general. La industria forestal comenzó a adquirir un mayor protagonismo dentro de la producción nacional, basado en la plantación de especies exóticas de rápido crecimiento, el fomento de las exportaciones y la concentración de los beneficios en manos de unas pocas familias de empresarios.

El sector forestal chileno ha consolidado un sostenido "éxito" en el plano macroeconómico, incrementando continuamente la producción, las exportaciones y las ganancias de las grandes empresas forestales (Reyes et al. 2014), convirtiéndose en el segundo sector económico más importante del país detrás de la gran minería del cobre. Sin embargo, este crecimiento se basa en precarias condiciones sociales y laborales para los obreros, así como en acumulativos impactos ecológicos, los que a su vez afectan

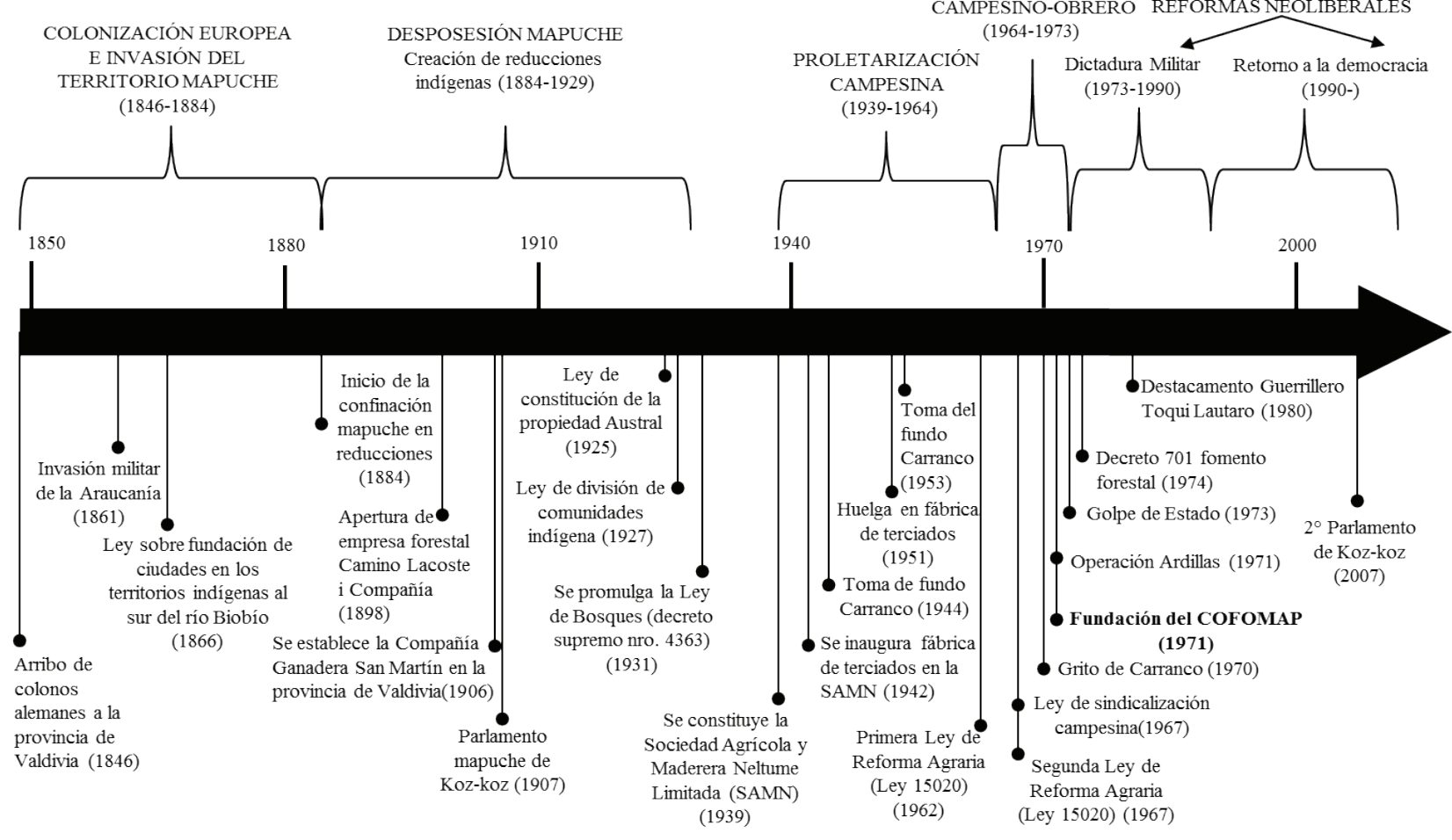

Figura 2. Principales acontecimientos vinculados a la historia del COFOMAP.

Milestones in the history of Panguipulli Forestry and Timber Complex. 
negativamente la sustentabilidad de los medios de vida de la población rural (Frêne y Núñez 2010).

De acuerdo con Frêne y Núñez (2010), dentro de la gran industria de la madera el $55 \%$ de los ingresos los reciben los accionistas de grupos económicos, mientras que solo un $22 \%$ es capturado por los obreros menos calificados, de los cuales menos del $30 \%$ cuenta con contrato permanente y más del $80 \%$ se encuentra bajo la línea de la pobreza. Consecuentemente, las regiones de Chile donde se concentra la actividad forestal muestran los peores índices de desarrollo humano y pobreza a escala nacional (Donoso y Otero 2005, Frêne y Núñez 2010). El abuso y explotación de los obreros, ha gatillado también tomas y huelgas. En mayo de 2007, en medio de una manifestación iniciada por los trabajadores subcontratados de la empresa Bosques Arauco S.A., muere a raíz de varios disparos policiales, Rodrigo Cisterna, obrero forestal de 26 años. Los trabajadores llevaban meses movilizados reclamando mejoras salariales y laborales, sin lograr acuerdo con la empresa de propiedad de la familia Angelini, uno de los grupos económicos más poderosos del país, poseedor de un patrimonio de más de 1.610 .000 hectáreas de terreno (Frêne y Núñez 2010). Tras la muerte de Rodrigo, el petitorio de demandas de los trabajadores es aceptado. Seis años más tarde, la justicia determinó la responsabilidad del Estado de Chile en la muerte del obrero.

Los impactos sobre el paisaje y la provisión de servicios ecosistémicos provocados por el actual modelo de desarrollo forestal, han sido también descritos en la literatura científica (Aguayo et al. 2009, Armesto et al. 2010, Lara et al. 2012, Miranda et al. 2015). El reemplazo de bosque nativo por plantaciones forestales exóticas ha sido asociado con una disminución en la disponibilidad de agua en grandes y pequeñas cuencas de la zona Centro-sur de Chile (Lara et al. 2009, Little et al. 2009), lo que genera también conflictos entre las comunidades locales, las empresas y el Estado (Frêne et al. 2014).

Actualmente, las comunidades mapuche ubicadas en la zona de desarrollo del COFOMAP, se mantienen articuladas en torno a la defensa de su territorio frente a un creciente número de proyectos hidroeléctricos impulsados por el Estado y empresas privadas (Morales 2009, Pino y Cardyn 2014). En 2007, cien años después de la reunión original, los Lonkos mapuche de la precordillera de Valdivia celebraron un nuevo parlamento en el valle de Koz-koz. Al igual que en 1907, la dominación ejercida por el Estado, junto con las acciones destructivas realizadas por parte de la sociedad chilena contra el pueblo mapuche, fueron temas relevantes en este nuevo parlamento (Morales 2009).

La experiencia del COFOMAP constituye el vuelco de las relaciones de poder dentro de la producción forestal y maderera, con los obreros forestales y campesinos como controladores activos en el uso del bosque y la repartición de los beneficios económicos y sociales derivados de él. Las estructuras de dominación ejercidas a través de décadas por los dueños de la tierra y medios de producción, fueron suprimidas mediante una alianza en la que participaron Estado, obreros, técnicos y profesionales. Si bien, la política de reivindicación social y económica de los trabajadores impulsada durante la Unidad Popular influyó notablemente en las tomas de fundos que dieron origen al COFOMAP, los obreros rurales, campesinos y mapuche, llevaban décadas intentando cambiar aspectos estructurales de la producción forestal y maderera, tal como ha sido mostrado en este estudio (figura 2). La importancia del COFOMAP, radica entonces en la materialización de esos cambios en favor de los intereses perseguidos por la clase obrera. Su período de funcionamiento es recordado como un período de abundancia por las comunidades locales y su historia ha influido en la memoria e identidad local de los habitantes de Neltume y Panguipulli (Skewes et al. 2011, Pino y Cardyn 2014).

En el contexto actual de crisis social y ecológica, diversos enfoques promueven formas de comanejo en el uso de los recursos naturales, los cuales involucran la participación de los usuarios directos en la toma de decisiones como una manera de alcanzar tanto objetivos de sustentabilidad ecológica y justicia social. No obstante, en la práctica, la participación queda supeditada al poder político de los actores involucrados, que a su vez se configura a través del funcionamiento de las instituciones sociales. En ese sentido, los autores creen que el COFOMAP, como experiencia de comanejo del bosque, aporta elementos para una revisión crítica de estos enfoques, muchas veces carentes de una perspectiva política, expandiendo el debate hacia aspectos estructurales de la producción forestal, como la concentración de la tierra y las ganancias, los intereses que apoya y promueve el Estado a través de las políticas públicas, y la configuración de las relaciones de poder entre actores involucrados en la producción maderera y forestal.

\section{AGRADECIMIENTOS}

Este trabajo está dedicado a la memoria del profesor de historia, geografía y educación cívica Ricardo Rivas Santana. El autor y las autoras agradecen especialmente a los dos informantes entrevistados, quienes entregaron aportes fundamentales para la generación de este artículo. Así mismo, se agradece al Museo y Memoria Neltume por la entrega de información tanto en forma de relato como cartográfica. Finalmente, agradecemos los valiosos comentarios y sugerencias entregadas por el editor y comité editorial de revista Bosque, así como también por parte de los dos árbitros anónimos que revisaron este artículo.

\section{REFERENCIAS}

Aguayo M, A Pauchard, G Azócar, O Parra. 2009. Land use change in the south central Chile at the end of the $20^{\text {th }}$ century. Understanding the spatio-temporal dynamics of the landscape. Revista Chilena de Historia Natural 82: 361-374.

Almonacid F. 2008. La división de las comunidades indígenas 
del sur de Chile, 1925-1958: Un proyecto inconcluso. Revista de Indias 68(243): 115-150.

Almonacid F. 2009. El Problema de la Propiedad de la Tierra en el Sur de Chile (1850-1930). Revista Historia 42: 5-56.

Anderson C, G Likens, R Rozzi, J Gutiérrez, J Armesto, A Poole. 2008. Integrating science and society through long-term socio-ecological research. Environmental Ethics 30(3): 295-312.

Armesto J, D Manuschevich, A Mora, C Smith-Ramírez, R Rozzi, AM Abarzúa, PA Marquet. 2010. From the Holocene to the Anthropocene: A historical framework for land cover change in southwestern South America in the past 15,000 years. Land Use Policy 27: 148-160.

Aylwin J. 2002. El derecho de los pueblos indígenas a la tierra y al territorio en América Latina: Antecedentes históricos y tendencias actuales. In Sesión del grupo de trabajo sobre la sección Quinta del Proyecto de Declaración con especial énfasis en las formas tradicionales de propiedad y supervivencia cultural. Derecho a tierras y territorios. Consejo Permanente de la Organización de Estados Americanos - OEA (Comisión de Asuntos Jurídicos y Políticos) eds. Washington D.C. EE.UU. 34 p.

Bellisario A. 2013. La reforma agraria chilena. Reformismo, socialismo y neoliberalismo, 1964-1980. Historia Agraria 59: $159-190$.

Bengoa J. 2000. Historia del pueblo mapuche: siglo XIX y XX. Santiago, Chile. LOM Ediciones. 430 p.

Bengoa J. 2007. El tratado de Quilín. Documentos adicionales a la historia de los antiguos mapuches del Sur. Santiago, Chile. Catalonia. 132 p.

Bize C. 2012. Historia y memoria hacia una experiencia de poder popular: Los trabajadores de la madera en la cordillera de Valdivia. (Neltume, 1970 - 1971). In Piper I, B Rojas eds. Memorias, Historias y Derechos Humanos. Santiago, Chile. Programa Domeyko Sociedad y Equidad, Universidad de Chile. 179 p.

Bravo J. 2012. De Carranco a Carrán. Las tomas que cambiaron la historia. Santiago, Chile. LOM Ediciones. 268 p.

Camus P, ME Solari. 2008. La invención de la selva austral. Bosque y tierras despejadas en la cuenca del río Valdivia (siglos XVI-XIX). Revista de Geografia Norte Grande 40: 5-22.

Cardyn P. 2006. Pisadas de Río Monte. Estampas de la Cordillera y del Complejo Maderero Panguipulli. Panguipulli, Chile. Pedro Cardyn ed.

Castells M. 2009. Communication power. New York, United States. Oxford University Press. 608 p.

Chacón I. 1994. Políticas forestales en Chile: 1931-1992. Revista Universum (año 9): 113-131.

Chonchol J. 1994. Sistemas agrarios en América Latina: de la etapa prehispánica a la modernización conservadora. México DF, México. Fondo de Cultura Económica. 177 p.

CODEPU (Corporación de Promoción y Defensa de los Derechos del Pueblo, CL). 1991. Chile: Recuerdos de la Guerra. Valdivia - Neltume - Chihuio - Liquiñe (vol. 2), de la Serie Verdad y Justicia. Santiago. Chile. CODEPU. 182 p.

Comité Memoria de Neltume. 2003. Guerrilla en Neltume: una historia de lucha y resistencia en el sur chileno. Santiago, Chile. LOM Ediciones. 320 p.

Contreras A, L Otero, L Barrales, I Ojeda. 1996. Estudio de crecimiento de una plantación de raulí ubicada en el sector de Panguipulli y expectativas de crecimiento. Bosque 17(1): 3-7.
Correa M, E Mella. 2010. Las razones del illkun/enojo. Memoria, despojo y criminalización el en territorio mapuche. Santiago, Chile. Observatorio Derechos de los Pueblos Indígenas. LOM Ediciones. $322 \mathrm{p}$.

Correa M, R Molina, N Yáñez. 2005. La reforma agraria y las tierras mapuches: Chile 1962-1975. Santiago, Chile. LOM Ediciones. 470 p.

Díaz A. 2005. Parlamento de Coz - Coz: breve relación del parlamento mapuche de $\mathrm{Coz}-\mathrm{Coz}$ realizado el 18 de enero de 1907. Panguipulli, Chile. Araucaria. 198 p.

Di Giminiani P. 2015. Being from the land: Memory, self and the power of place in indigenous Southern Chile. Ethnos: 1-25. DOI: $10.1080 / 00141844.2015 .1028566$.

Domeyko I, M Förster. 1850. Memoria sobre la colonización en Chile. Consultado 15 nov. 2014. Disponible en http://www. memoriachilena.cl/archivos2/pdfs/MC0001428.pdf

Donoso PJ, L Otero. 2005. Hacia una definición de país forestal: ¿Dónde se sitúa Chile? Bosque 26(3): 5-18.

Foerster R. 1996. La propiedad Huilliche en los llanos de Valdivia y Río Bueno. In Vergara J, A Mascarreño, R Foerster eds. La propiedad Huilliche en la provincia de Valdivia. Santiago, Chile. Corporación Nacional de Desarrollo Indígena. $374 \mathrm{p}$.

Frêne C, M Núñez. 2010. Hacia un nuevo Modelo Forestal en Chile. Revista Bosque Nativo 47: 25-35.

Frêne C, G Ojeda, J Santibáñez, C Donoso, J Sanzana, C Molina, P Andrade, M Núñez-Ávila. 2014. Agua en Chile: Diagnósticos territoriales y propuestas para enfrentar la crisis hídrica en Chile. Santiago, Chile. Imprenta América. 60 p.

Galilea S, J Leyton, F Ordóñez, F Salamanca. 1972. Agentes políticos y reestructuración del espacio y la producción de una región de Chile. Revista de Estudios Urbanos Regionales 2(4): 67-82.

Gómez S. 2002. Organización campesina en Chile: reflexiones sobre su debilidad actual. Revista Austral de Ciencias Sociales 6: 3-18.

Hauck J, C Stein, E Schiffer, M Vandewalle. 2015. Seeing the forest and the trees: Facilitating participatory network planning in environmental governance. Global Environmental Change 35: 400-410.

Herrmann T. 2006. Indigenous knowledge and management of Araucaria araucana forest in the Chilean Andes: implications for native forest conservation. Biodiversity and Conservation 15:647-662.

Kangas A, S Laukkanen, J Kangas. 2006. Social choice theory and its applications in sustainable forest management $-\mathrm{a}$ review. Forest Policy and Economics 9: 77-92.

Kay C. 1974. La participación campesina bajo el gobierno de la U. P. (Unidad Popular, Chile). Revista Mexicana de Sociología 36(2): 279-295.

Kay C. 1977. Tipos de reforma agraria y sus contradicciones: el caso de Chile. Revista Mexicana de Sociología 39(3): 857-872.

Kay C., M Pineda. 1998. ¿El fin de la reforma agraria en América Latina? El legado de la reforma agraria y el asunto no resuelto de la tierra. Revista Mexicana de Sociología 60(4): 63-98.

Klein E. 1973. Conflict between rural workers and landowners in central Chile. PhD thesis. Brighton, UK. University of Sussex. 308 p.

Klubock TM. 2004. Labor, land, and environmental change in the forestry sector in Chile, 1973-1998. In Winn P ed. Vic- 
tims of the Chilean miracle. Workers and neoliberalism in the Pinochet Era, 1973-2003. Durham, USA. Duke University Press. 429 p.

Lara A, C Little, R Urrutia, J McPhee, C Álvarez-Garretón, C Oyarzún, D Soto, P Donoso, L Nahuelhual, M Pino, I Arismendi. 2009. Assessment of ecosystem services as an opportunity for the conservation and management of native forests in Chile. Forest Ecology and Management 258: 415-424.

Lara A, ME Solari, MDR Prieto, MP Peña. 2012. Reconstruction of vegetation cover and land use $c a$. 1550 and their change towards 2007 in the Valdivian Rainforest Ecoregion of Chile $\left(35^{\circ}-43^{\circ} 30^{\prime} \mathrm{S}\right)$. Bosque 33(1): 13-23.

Le Bonniec F. 2013. La participación de las comunidades mapuche-huilliche en el proceso de la Reforma Agraria en la Provincia de Valdivia (1970-1973). Revista Austral de Ciencias Sociales 24: 27-49.

Little C, A Lara, J McPhee, R Urrutia. 2009. Revealing the impact of forest exotic plantations on water yield in large escale watersheds in South-Central Chile. Journal of Hydro$\log$ 274: 162-170.

Marimán J. 2012. Autodeterminación: Ideas políticas mapuche en el albor del siglo XXI. Santiago, Chile. LOM Ediciones. $358 \mathrm{p}$.

Miranda A, A Altamirano, L Cayuela, F Pincheira, A Lara. 2015. Different times, same story: Native forest loss and landscape homogenization in three physiographical areas of southcentral of Chile. Applied Geography 60: 20-28.

Moore JW. 2003. The Modern World-System as environmental history? Ecology and the rise of capitalism. Theory and Society 32(3): 307-377.

Morales R. 2009. Resonancias políticas de una protesta centenaria: Koyautun Mapunche Williche, Koz Koz Mapu Kuifi Kiñe Pataka Tripantu. Revista Austral de Ciencias Sociales 15: 25-40.

Morales E, J Oltremaría, A Norambuena, G Kunstman, F Saelzer, B Olivares, L Salinas, O Oyarzún, R Jara, E Fried. 1977. Sector Forestal - Provincia de Valdivia. Bosque 2(1): 45-59. Ostrom E. 2009. A general framework for analyzing sustainabili- ty of social-ecological systems. Science 325: 419-422.

Paletto A, K Hamunen, I De Meo. 2015. Social network analysis to support stakeholder analysis in participatory forest planning. Society \& Natural Resources 28: 1108-1125.

Pino A, P Cardyn, Grupo de Trabajo Panguipulli. 2014. La reserva de la Biosfera de los Bosques Templados Lluviosos de los Andes Australes y las singularidades territoriales de la comuna de Panguipulli. In Moreira-Muñoz A, A Borsdorf eds. Reservas de la Biosfera de Chile: Laboratorios para la Sustentabilidad. Axams, Austria. Academia de Ciencias Austríaca, Pontificia Universidad Católica de Chile, Instituto de Geografía (Santiago de Chile), Serie Geolibros n 17. 332 p.

Reyes R, C Sepúlveda, L Astorga. 2014. Gobernanza del sector forestal chileno. Tensiones y conflictos entre las fuerzas del mercado y las demandas de la ciudadanía. In Donoso C, M González, A Lara eds. Ecología forestal. Bases para el manejo sustentable y conservación de los bosques nativos de Chile. Valdivia, Chile. Ediciones Universidad Austral de Chile. 720 p.

Rivas R. 2006. Desarrollo forestal de Neltume; Estado y trabajadores. (1924-1990). Tesis de licenciado en historia. Valdivia, Chile. Facultad de Filosofía y Humanidades, Universidad Austral de Chile. 85 p.

Solari ME, C Cueto, F Hernández, JF Rojas, P Camus. 2011. Procesos territoriales y bosques en la cuenca del río Valdivia (siglos XVI-XIX). Revista de Geografia Norte Grande 49: 45-62.

Skewes JC, D Guerra, P Rojas, MA Mellado. 2011. ¿La memoria de los paisajes o los paisajes de la memoria? Los enigmas de la sustentabilidad socioambiental en las geografías en disputa. Desenvolvimento e Meio Ambiente 23: 39-57.

Thiesenhusen W. 1966. Agrarian reform and economic development in Chile: Some cases of colonization. Land Economics 42(3): 282-292.

Unidad Popular. 1970. Programa básico de gobierno de la Unidad Popular: candidatura presidencial de Salvador Allende. Santiago, Chile. Biblioteca Nacional de Chile. 47 p.

Vega M. 1958. El aporte alemán al progreso de Chile. Anales de la Universidad de Chile 109-110: 488-495. 